\title{
Pushing the Limits of Luminescence Thermometry: Probing the Temperature of Proteins in Cells
}

\author{
Glauco Maciel ${ }^{1}$ \\ ${ }^{1}$ Universidade Federal Fluminense
}

July 22,2021

\begin{abstract}
Proteins are involved in numerous cellular activities such as transport and catalysis. Misfolding during biosynthesis and malfunctioning as a molecular machine may lead to physiological disorders and metabolic problems. Protein folding and mechanical work may be viewed as thermodynamic energetically favorable processes in which stochastic nonequilibrium intermediate states may be present with conditions such as thermal fluctuations. In my opinion, measuring those thermal fluctuations may be a way to access the energy exchange between the protein and the physiological environment and to better understand how those nonequilibrium states may influence the misfolding/folding process and the efficiency of the molecular engine cycle. Here, I discuss luminescence thermometry as a possible way to measure those temperature fluctuations from a single molecule experimental perspective with its current technical limitations and challenges.
\end{abstract}

\section{Pushing the Limits of Luminescence Thermometry: Probing the Temperature of Proteins in Cells}

Glauco S. Maciel11E-mail: gsmaciel@id.uff.br ORCID: http://orcid.org/0000-0002-2221-198X

Instituto de Física, Universidade Federal Fluminense, 24210-346, Niterói, RJ, Brazil.

\begin{abstract}
Proteins are involved in numerous cellular activities such as transport and catalysis. Misfolding during biosynthesis and malfunctioning as a molecular machine may lead to physiological disorders and metabolic problems. Protein folding and mechanical work may be viewed as thermodynamic energetically favorable processes in which stochastic nonequilibrium intermediate states may be present with conditions such as thermal fluctuations. In my opinion, measuring those thermal fluctuations may be a way to access the energy exchange between the protein and the physiological environment and to better understand how those nonequilibrium states may influence the misfolding/folding process and the efficiency of the molecular engine cycle. Here, I discuss luminescence thermometry as a possible way to measure those temperature fluctuations from a single molecule experimental perspective with its current technical limitations and challenges.
\end{abstract}

Keywords: thermometry, proteins, thermodynamics, luminescence, nanoparticles.

\section{Introduction}

Temperature is a fundamental physical parameter that can be defined thermodynamically as the degree of kinetic energy of the constituents of the matter. Macroscopically, temperature is generally associated with heat and its measurement has been commonly used to monitor the proper operation of electrical, mechanical, chemical, and biological systems. Luminescence thermometers rely on the change of the luminescence intensity, bandwidth, peak wavelength, or lifetime, with the temperature. Luminescence thermometers are particularly useful for fast readout where adverse conditions are found. For example, temperature monitoring in electric 
power transformer networks, where quick rise of oil temperature and the presence of high magnetic fields limits the use of conventional thermocouples. As we moved towards miniaturization, nanoscale luminescence thermometers have been developed for use in, for example, studies of temperature gradients in electronic integrated circuitry, microfluidic devices, and intracellular structures. ${ }^{1}$ Recently, studies of thermogenesis in organelles such as mitochondria, plasmatic reticulum, centrosome, and nucleus using luminescence-based thermometers have been reviewed. ${ }^{2}$ Intracellular metabolic processes are diverse and very complex with the regulation of the biochemical reactions being carried out mainly by enzymatic activity. Understanding the mechanisms behind heat production in cells on a molecular level is a challenge. In this scenario, luminescence nanothermometers may be a suitable tool to probe temperature gradients, especially in proteins.

\section{Protein in a cell: a thermodynamic approach}

Proteins participate in different cellular functions such as catalysis, structure, and transport. ${ }^{3}$ Proteins are synthesized on ribosomes and the polypeptide linear chain folds spontaneously into a three-dimensional structure known as the native state. The amino acid sequence that is characteristic of each type of protein is responsible for the folding dynamics and the morphology of the three-dimensional folded state. Proteins may be unfolded by an external perturbation (physical, chemical, or mechanical agent) but they generally spontaneously return to the folded native state in physiological environments. Protein folding may be viewed as a thermodynamic process. The entire folding process is very complex with numerous possible stochastic thermodynamic pathways and meta-stable intermediate states that leads to a minimum Gibbs free energy equilibrium state, according to the folding energy funnel hypothesis. Figure 1 shows the energy landscape of a protein during folding. The width of the dominant funnel represents the configurational entropy while the depth represents the free energy of an individual configuration averaged over the solvent alone. After the partially ordered molten globule is reached, another appropriate reaction coordinate to describe the folding process is $Q$, the fraction of native contacts. In the upper region of the funnel, helical order is established and the protein becomes compact. ${ }^{4}$ 


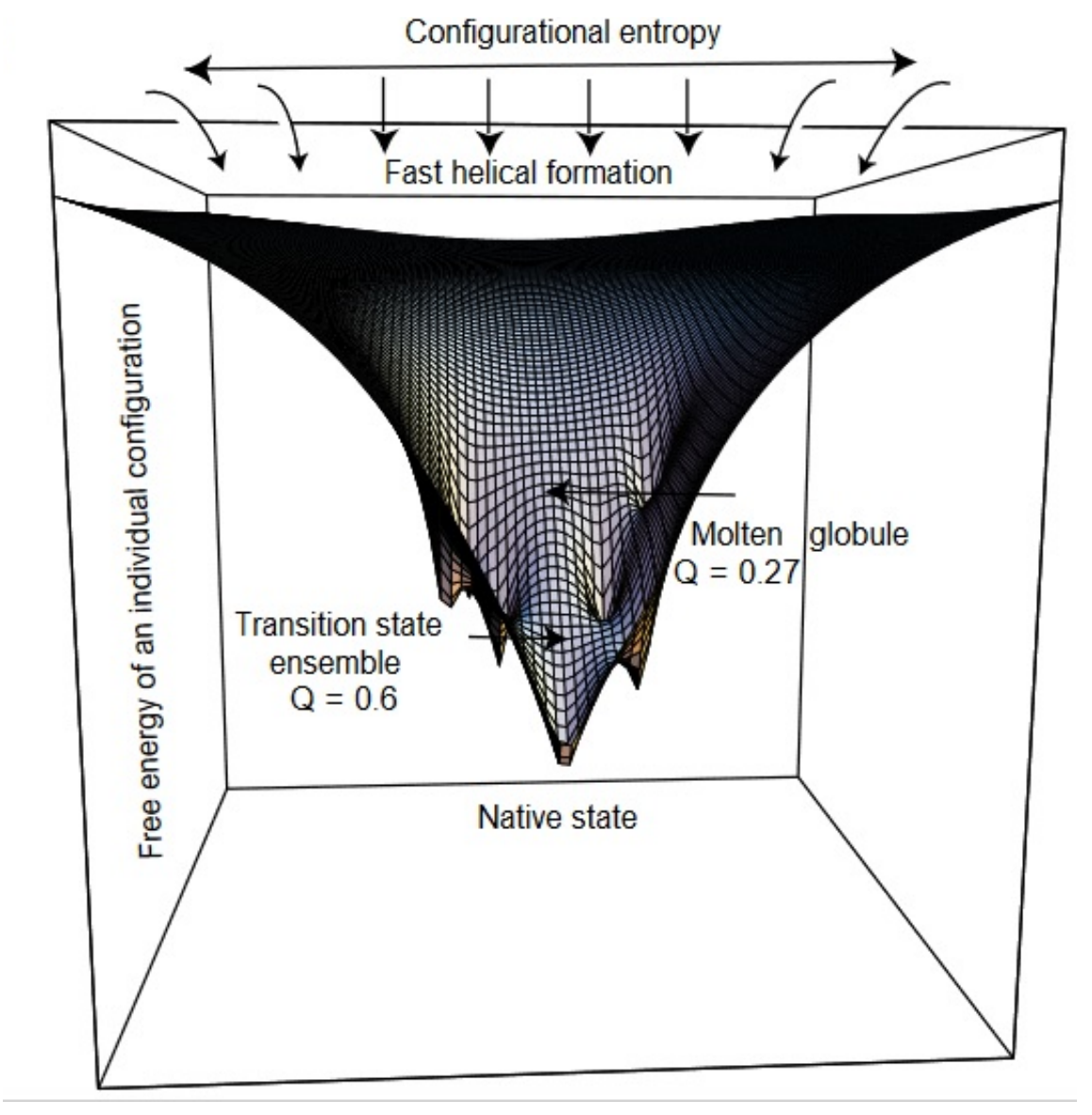

Figure 1. The protein folding funnel for a fast-folding helical protein. Copyright Elsevier Cell Press 1996.

While the difference in the Gibbs free energy, $\Delta \mathrm{G}$, between the initial and the final thermodynamic equilibrium states of the unfolding/refolding mechanisms has been investigated from both a theoretical and experimental points of view, ${ }^{5}$ the study of intermediate nonequilibrium states is also important as these states may influence the folding process during biosynthesis and may help understand misfolding, which is the cause of some diseases. ${ }^{6}$ Thermodynamically, the folding process might occur at constant temperature (the physiological environment is assumed to be a thermal bath) but stochastic nonequilibrium intermediate states may present thermal fluctuations that may be related to energy (heat) exchange between the protein and the physiological environment. ${ }^{7}$ Therefore, the measurement of those temperature fluctuations/heat exchange is important to better understand the thermodynamics of those intermediate nonequilibrium states and its influence during the (mis)folding process. Besides the folding process, real time temperature measurements would also be appealing for the study of fluctuations due to thermal noise in proteins that work as motors (rotary and linear engines) and shuttles. ${ }^{8}$ These proteins generally use chemical reactions such as ATP hydrolysis as a fuel to generate mechanical work. Taking into account the first law of thermodynamics, part of the energy received by the protein is converted into useful work and the remaining energy is lost as heat. When proteins help transporting ions/molecules through cell membrane, for example, the work is considered thermodynamically favorable when the transport is performed from a region of high ion $/ \mathrm{molecule}$ concentration to a region of low ion/molecule concentration. In this case, $\Delta \mathrm{G}$ is associated to the chemical potential difference and the reversible work needed to perform the transport of ions/molecules across the membrane. ${ }^{9}$ As a transport phenomenon, it may also be viewed as a nonequilibrium thermodynamical process in which heat conduction may lead to gradients of temperature and entropy production with time. Thus, the direct measurement of those temperature gradients may be relevant information to assess and perhaps 
optimize the efficiency of the energy cycle of the molecular motor.

\section{Protein dynamics: theoretical and experimental analyses}

Protein dynamics has been investigated by a number of theoretical methodologies and experimental techniques. From a theoretical point of view, computational and modeling have been using to analyze energy change in protein folding using statistical mechanics and nonequilibrium thermodynamics. ${ }^{10,11}$ From the experimental point of view, X-ray crystallography, optical spectroscopy, and nuclear magnetic resonance have been commonly used. ${ }^{12}$ All these experimental techniques are employed in ensembles, i.e., the information retrieved is based on the collective behavior (average) of the objects of study, and therefore individual behavior associated to stochastic nonequilibrium fluctuations are not detectable. Single molecule experiments have been focused on fluorescence correlation spectroscopy to study protein folding as a diffusive process on a free-energy surface ${ }^{13}$ and optical tweezer/atomic force microscopy to establish mechanical forces to stretch (unfold) the macromolecule and observe the dynamics of the refolding process. ${ }^{14}$ Concerning proteins working as motors and shuttles, experimental techniques such as magnetic tweezers and electrorotation have been used to estimate the thermodynamic efficiency under some constraint conditions such as quasi static limit. ${ }^{15}$ All the single molecule experiments mentioned here were based on an external stimulus to induce some mechanical motion (folding, rotation) in order to study the dynamics of the system. However, biological systems under external stimulation and without external stimulation may undergo different metabolisms. Nothing has been tried concerning real time temperature measurements on individual proteins spontaneously folding/performing a mechanical work. As mentioned earlier in the text, valuable information about misfolding during biosynthesis and poor engine efficiency, both possibly associated to stochastic thermodynamical nonequilibrium states, may be hypothetically retrieved by measurements of thermal fluctuations and, consequently, estimates of energy related thermodynamic variables. Actually, the study of temperature fluctuations in nonequilibrium thermodynamical phenomena can be more widespread, not restricted only to proteins but it can be extended to other types of biomacromolecules, such as for example DNA. Thus, the potential for using nanothermometers for probing nonequilibrium phenomena in biochemical reactions involving macromolecules is substantial.

\section{Luminescence thermometry in a single protein: the experimental limitations and challenges}

Optical temperature sensing using a single luminescent object linked to a biomacromolecule presents many challenges. For accurate real time temperature monitoring of biomacromolecules, the luminescence nanothermometer has to be in contact with the biomacromolecule while the temperature is estimated through some remote detection system that records some change of the luminescence profile with the temperature on the object of study. Imaging and sensing of biological ensemble systems have been accomplished using different classes of luminophores, such as semiconductor quantum dots, lanthanide doped nanocrystals, and fluorescent proteins. ${ }^{16}$ Inorganic compounds have better photostability, i.e., they do not undergo photodegradation, a characteristic of organic compounds, and therefore they are more convenient for single molecule studies. Due to its lower toxicity level, lanthanide doped nanocrystals are generally more appropriate than semiconductor quantum dots, which contains elements such as Cd, Se and S. However, due to its larger size and constitution, inorganic nanoparticles-biomacromolecules conjugates are much more complicated to fabricate than fluorescent proteins-biomacromolecules conjugates. ${ }^{16}$ Thermometry using a single fluorescent protein is complicated by the fact that besides photodegradation due to long exposure to light excitation, organic molecules also show blinking, a characteristic of electronic population of dark states during relaxation. Lanthanide doped nanoparticles, on the other hand, do not present blinking because a single nanoparticle has many luminophores, the lanthanide ions. Recent imaging and spectral analysis of the luminescence profile of individual lanthanide doped nanoparticles are available in literature. ${ }^{17,18}$ Figure 2 shows the luminescence spectral profile of a $\mathrm{NaYF}_{4}$ nanoparticle doped lanthanides $\left(\mathrm{Yb}^{3+}\right.$ and $\left.\mathrm{Er}^{3+}\right)$. The signal was recorded with a scanning confocal microscopy set-up. ${ }^{17}$ Observe that the relative intensity of the two emission bands at $520-535 \mathrm{~nm}$ and $540-560 \mathrm{~nm}$, which are radiative relaxations from thermally coupled electronic states ${ }^{4} \mathrm{~S}_{3 / 2}$ and ${ }^{2} \mathrm{H}_{11 / 2}$ of $\mathrm{Er}^{3+}$, changes with the temperature in a way that optical temperature sensing is feasible. 


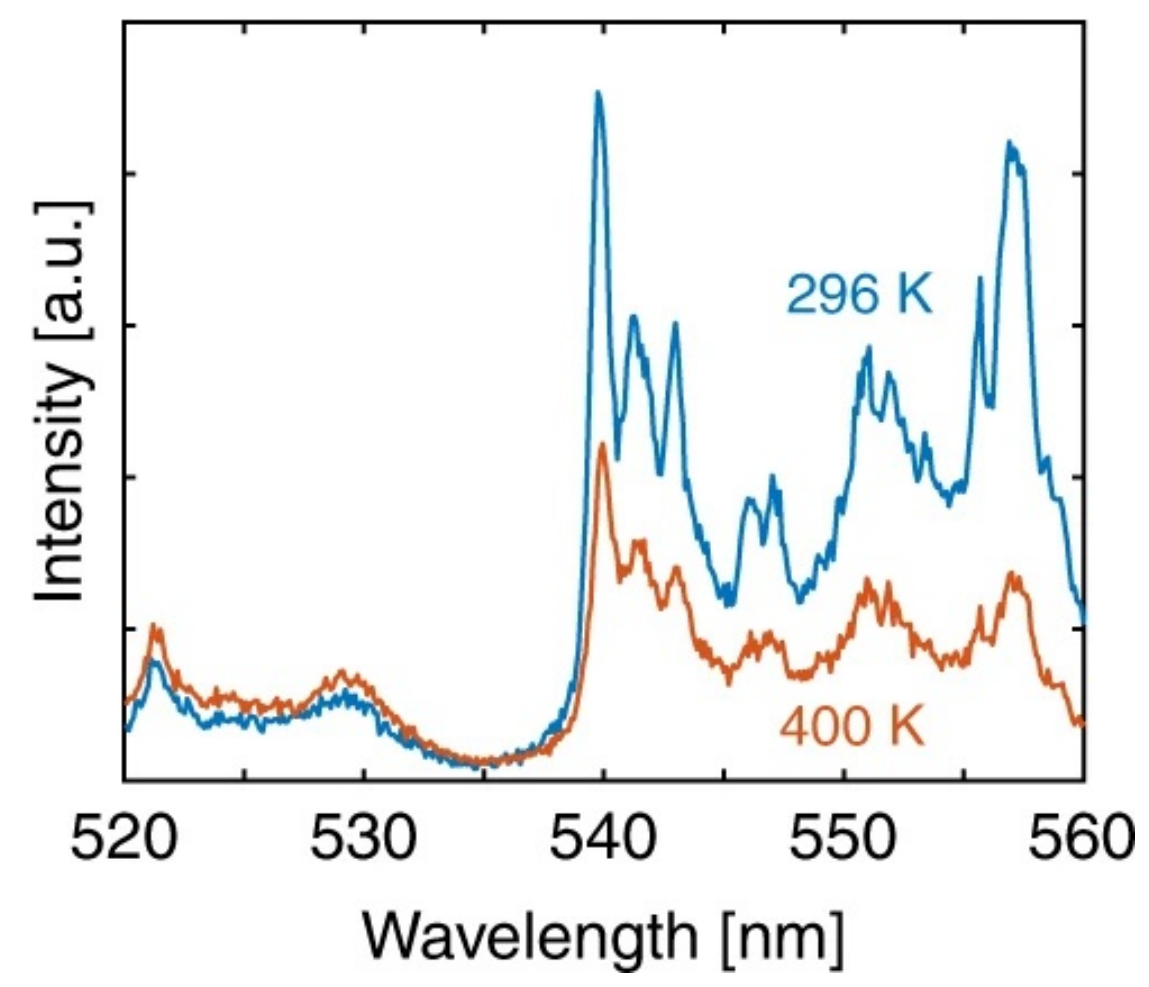

Figure 2. Luminescence intensity thermometry measurements of an individual lanthanide doped nanoparticle. Representative spectra taken at two temperatures. Copyright Springer Nature 2018.

Single molecule spectroscopy on nanoparticle-protein conjugate has not been reported so far. However, conjugation of proteins with nanoparticles has been proposed. For example, Figure 3 shows an image of a space-filling modeling approach to study interactions between a maltose-binding protein and a semiconductor quantum dot, ${ }^{19}$ with surface sulfur atoms in teal and zinc atoms in pink. The red shell shows the estimated outer radius of the dihydrolipoic acid ligand. The side chain of Lys-370 is shown in blue.

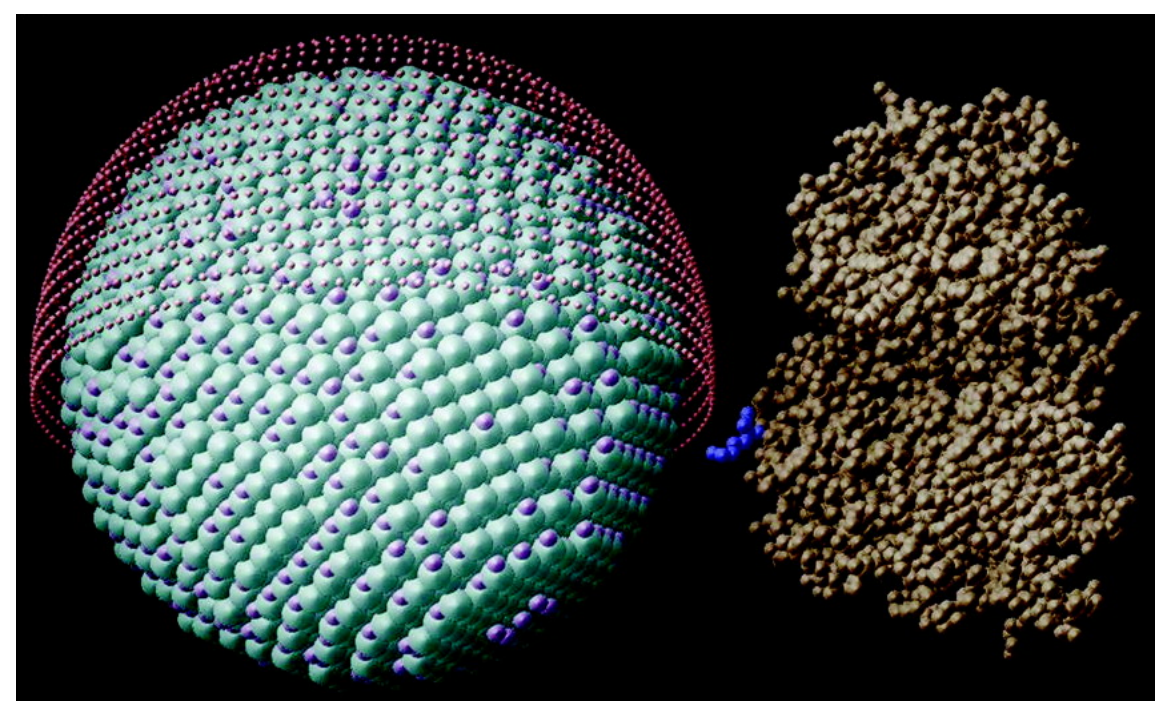


Figure 3. Protein-quantum dot bioconjugate. Copyright National Academy of Sciences of the United States of America 2004.

It has been discussed that the structure and function of the protein can be influenced by many factors such as the size of the nanoparticle, the nanoparticle ligand, the material of the nanoparticle, the stoichiometry of the conjugate, the labeling site of the protein and the nature of the linkage. ${ }^{20}$ Folded proteins have an average size of a few nanometers (typically a diameter of 5-10 nm). Thus, ideally, the nanoparticle should be much smaller than that to avoid physical interference over the morphological dynamics of the protein. Up to now, the smallest reported synthesized nanoparticle has a size of a few nm. Another characteristic that should be avoided is fluorescence resonance energy transfer (FRET), in which energy migrates from the excited luminescent temperature probe to the conjugated protein. FRET must be avoided as (a) it may decrease the intensity of the luminescence from the nanoparticle, which is used for thermometry, and (b) it may induce change of the protein energy state. Additionally, direct photon excitation of the protein must be avoided. As the nanoparticle-protein conjugates are much smaller than the spatial resolution of the optical system, typically larger than a few hundred nanometers (the Rayleigh diffraction limit), photons of the excitation source strike both the nanoparticle and protein. Therefore, excitation sources have to operate at wavelengths outside the absorption bands of electronic transitions of the organic structure, the so-called biological windows. Another important point that should be taken into consideration is the excitation power density. If the excitation power density is too high, the reading of temperature may be compromised by optically induced heating caused by strong absorption of light by the nanoparticle. A possible solution is the use of very small excitation powder densities, which besides avoiding optical heating also prevents nonlinear optical processes such as multi-photon absorption in both the nanoparticle and the protein. As the luminescence intensity is proportional to the excitation power density and the amount of luminophores, the intensity of the luminescence is expected to be very low for a single nanoparticle. A mechanism called photon avalanche have been proposed as a way to improve imaging resolution and brightness at a single nanoparticle level. ${ }^{18}$ For thermometry application, fundamental parameters are the absolute temperature sensitivity S, defined as the ratio in which the thermometric parameter changes with temperature, and the uncertainty in temperature $\delta \mathrm{T} \cdot{ }^{21}$ To detect small thermal fluctuations, as should be the case found at a molecular level, S has to be very high while $\delta \mathrm{T}$ has to be extremally small. Engineering nanomaterials with tailored structural and bonding characteristics helps improve S. For example, luminescence thermometry has been studied using the luminescence intensity ratio (LIR) technique. ${ }^{21}$ Assuming a Boltzmann population distribution among two thermally coupled electronic states (TCES) of lanthanide ions in solids, it is observed that $\mathrm{S}$ is a function of the barycenter of the energy bandgap between the TCES. In this case, the choice of the host material is a key factor because the crystal field influences the spectral peak position of emission bands. The parameter $\delta \mathrm{T}$, on the other hand, is related to different factors such as, for example, the signal-to-noise ratio and integration time of the reading of the thermometric parameter. Thermal fluctuations related to nonequilibrium thermodynamic states are expected to be fast compared with other time scales of events happening during the protein activity and dynamics (microseconds to seconds). As a result, integration time of the reading of the thermometric parameter during the thermal fluctuation event has to be relatively short. Thus, the need of advanced instrument technology with superior spectral and temporal resolutions associated with high electronic noise reduction is fundamental to minimize $\delta \mathrm{T}$.

\section{Conclusion}

Proteins have a fundamental role in biological processes. The dynamics of such biochemical activities are complex and a complete understanding of nonequilibrium states is currently lacking. In this scenario, there must be a tremendous effort to overcome all the current limitations that restrains measurements of thermal fluctuations involved in nonequilibrium thermodynamic states present on the complex dynamics of proteins. However, the insight to be gained with those measurements will help better understand the mechanisms involved in the energy balance of important biochemical reactions and processes involved in the correct/incorrect operation of those key biomolecules. Luminescence thermometry is an attractivity option to probe protein activity. Despite our current experimental limitations, it is matter of time for the progress in technological tools and methods to achieve a level in which it will be feasible to unveil the details of the 
fascinating world of proteins at work, especially the thermodynamics of nonequilibrium states.

\section{Conflict of interest}

The author declares that no conflict of interest exists.

\section{Acknowledgements}

This work was partially supported by the Brazilian National Council of Technological and Scientific Development (CNPq). Grant number: 305452/2019-3.

\section{References}

1. Carlos, L.D. and Palacio, F. Thermometry at the Nanoscale: Techniques and Selected Applications . The Royal Society of Chemistry; 2016.

2. Okabe, K., Sakaguchi, R., Beini, S. and Kiyonaka, S. Intracellular thermometry with fluorescent sensors for thermal biology.Pflugers Arch - Eur. J. Physiol. 2018; 470: 717-731.

3. Buxbaum, E. Fundamentals of Protein Structure and Function . Springer; 2015.

4. Onuchic, J. N., Socci, N. D., Luthey-Schulten, Z., Wolynes, P. G. Protein folding funnels: the nature of the transition state ensemble.Fold. Des. 1996; 1: 441-450.

5. Fang, Y. Gibbs free energy formula for protein folding. in Thermodynamics - fundamentals and its application in science (ed. Morales-Rodriguez, R.) 47-82. IntechOpen; 2012.

6. Chiti, F. and Dobson, C.M. Protein misfolding, functional amyloid, and human disease. Annu. Rev. Biochem. 2006; 75: 333-66.

7. Toyabe, S. and Sano, M. Nonequilibrium Fluctuations in Biological Strands, Machines, and Cells. J. Phys. Soc. Jpn. 2015; 84: 102001.

8. van den Heuvel, M.G.L. and Dekker, C. Motor Proteins at Work for Nanotechnology. Science 2007; 317: $333-336$.

9. Gura, M., Golcuka, M., Yilmaza, S.Z and Taka, E. Thermodynamic first law efficiency of membrane proteins. J. Biomol. Struct. Dyn.2020; 38: 439-449.

10. Li, Z. and Scheraga, H.A. Monte Carlo-minimization approach to the multiple-minima problem in protein folding. Proc. Natl. Acad. Sci. USA 1987; 84: 6611-6615.

11. West, D.K., Olmsted, P.D. and Paci, E. Free energy for protein folding from nonequilibrium simulations using the Jarzynski equality. J. Chem. Phys. 2006; 125: 204910.

12. Bartlett, A. I. and Radford, S. E. An expanding arsenal of experimental methods yields an explosion of insights into protein folding mechanisms. Nat. Struct. Mol. Biol. 2009; 16: 582-588.

13. Nettels, D., Hoffmann, A. and Schuler, B. Unfolded protein and peptide dynamics investigated with single-molecule FRET and correlation spectroscopy from picoseconds to seconds. J. Phys. Chem. B2008; 112: 6137-6146.

14. Ritort, F. Single-molecule experiments in biological physics: methods and applications. J. Phys.: Condens. Matter 2006; 18: R531.

15. Toyabe, S. and Muneyuki, E. Experimental thermodynamics of single molecular motor. Biophysics 2013; 9: 91-98.

16. Himmelstoß, S.F. and Hirsch, T. A critical comparison of lanthanide based upconversion nanoparticles to fluorescent proteins, semiconductor quantum dots, and carbon dots for use in optical sensing and imaging. Methods Appl. Fluoresc. 2019; 7: 022002.

17. Pickel, A.D., Teitelboim, A., Chan, E.M. et al. Apparent self-heating of individual upconverting nanoparticle thermometers. Nat. Commun. 2018; 9: 4907.

18. Lee, C., Xu, E.Z., Liu, Y. et al. Giant nonlinear optical responses from photon-avalanching nanoparticles. Nature 2021; 589: 230-235.

19. Medintz, I. L., Konnert, J. H., Clapp, A. R., Stanish, I., Twigg, M. E., Mattoussi, H., Mauro, J. M., Deschamps, J. R. A fluorescence resonance energy transfer-derived structure of a quantum dot-protein bioconjugate nanoassembly. Proc. Natl. Acad. Sci. USA 2004; 101: 9612-9617.

20. Aubin-Tam, M.E. and Hamad-Schifferli, K. Structure and function of nanoparticle-protein conjugates. 
Biomed. Mater. 2008; 3: 034001.

21. Brites, C.D.S., Balabhadra, S. and Carlos L.D. Lanthanide-based thermometers: at the cutting-edge of luminescence thermometry.Adv. Optical Mater. 2019; 7: 1801239. 